\title{
Dialogischer Pluralismus als Polit-Talk \\ - Ein Seminarkonzept zur Vermittlung \\ von Diskurskultur und angewandter Pluraler Ökonomik
}

\section{Elsa Egerer}

\section{Zusammenfassung}

Dialogische Kommunikation ist von zentraler Bedeutung für die Plurale Ökonomik. Der Beitrag verfolgt das Anliegen, eine, gute Diskurskultur' als Grundlage Pluraler Ökonomik zu stärken. Vor dem Hintergrund der sozialökologischen Krisen unserer Zeit wird zudem das Ziel einer anwendungsbezogenen, gestaltenden Pluralen Ökonomik erörtert. Das beschriebene innovative Vorgehen stellt einen Versuch dar, beide Aspekte in die Lehre zu integrieren. Zudem wird diskutiert, welche Herausforderungen sich didaktisch ergeben.

\section{Schlüsselbegriffe}

Plurale Ökonomik $\cdot$ Hochschuldidaktik $\cdot$ Dialog $\cdot$ Anwendungsbezug $\cdot$ Diskurskultur

Ich freue mich über Anregung und Kritik zum Beitrag. Bitte schreiben Sie mir dazu eine E-Mail an: elsa.egerer@gmail.com

E. Egerer $(\bowtie)$

Düsseldorf, Deutschland

E-Mail: elsa.egerer@googlemail.com

(C) Der/die Autor(en) 2021

J. Urban et al. (Hrsg.), Wirtschaft neu lehren, Sozioökonomische Bildung und Wissenschaft, https://doi.org/10.1007/978-3-658-30920-6_16 


\section{$1 \quad$ Entstehungskontext}

Plurale Ökonomik verspricht mehr Erkenntnisgewinn als eine monistische Sichtweise. Erfolgreiche Kommunikation ist dabei Voraussetzung, um die epistemologischen Früchte des Pluralismus zu ernten (Gräbner und Strunk 2018, S. 17). In diesem Sinne beschreiben diverse (Plurale) Ökonominnen und Ökonomen das Ziel eines ,interessierten“ (Dobusch und Kapeller 2012) bzw. „dialogischen“ Pluralismus (Rommel 2017). Als vielleicht prominenteste Fürsprecherin für das wirtschaftswissenschaftliche Gespräch sei an dieser Stelle McCloskey (1983, S. 483) zitiert, die sich intensiv mit der Rhetorik der Ökonomik auseinandersetzt, welche sie als das „Erforschen des Denkens durch Konversation" begreift. Ihr zufolge sollten sich Ökonominnen und Ökonomen ihrer Rhetorik bewusst werden, weil sich nur so die eigentlichen Gründe für Meinungsverschiedenheiten verstehen lassen. Hinsichtlich der ökonomischen Lehre äußert McCloskey:,,[...] economics is badly taught $[\ldots]$ because they [...] teach by axiom and proof instead of by problem-solving and practice" (ebd., S. 513).

Auch in diversen studentischen Forderungen nach mehr Pluralismus in der wirtschaftswissenschaftlichen Lehre findet sich das Bekenntnis zum dialogischen Pluralismus. So erachtet beispielsweise die International Student Initiative for Pluralism in Economics Pluralismus als geglückt, wenn eine „lebendige, intellektuell reichhaltige Debatte" angeregt wird (ISIPE 2014). Das Ziel, den „Diskurs innerhalb der Volkswirtschaftslehre“ fördern zu wollen, wird von diversen Gruppen auf lokaler Ebene betont (z. B. Plurale Ökonomik Mannheim e. V.). Dabei geht es den Studierenden nicht zuletzt um die Schaffung offener Räume und den Austausch auf Augenhöhe (z. B. Bündnis Transformative Lehre Halle 2017).

Die Kompetenz des dialogischen Pluralismus scheint unter Mainstreamökonominnen und Mainstreamökonomen wenig verbreitet, ist aber auch bei heterodoxen Ökonominnen und Ökonomen nicht immer anzutreffen. Vielleicht liegt hier ein Hauptgrund, wieso die Plurale Ökonomik hinter ihren Potenzialen zurückbleibt. Wenn der Diskurs jedoch eine essenzielle Rolle für die (Plurale) Ökonomik spielt, muss die Nachfolgegeneration der Ökonominnen und Ökonomen das wissenschaftliche und öffentliche Diskutieren lernen und üben, damit sich eine bessere Diskurskultur etablieren kann. Im Vordergrund steht dabei das Format, weniger der Inhalt wirtschaftswissenschaftlicher Lehre. 
Verschiedene Publikationen kritisieren des Weiteren eine Entkopplung der Wirtschaftswissenschaften von realweltlichen Fragen. Bereits im Jahr 1991 attestierte beispielsweise die Commission on Graduate Education in Economics der Ausbildung graduierter Ökonominnen und Ökonomen in den USA diesbezüglich Handlungsbedarf (Krueger et al. 1991). Bezeichnenderweise formuliert eine vom Institute for New Economic Thinking (INET 2010, S. 1) gegründete economics curriculum task force den Kern ihres Anliegens wie folgt: „Der zentrale Gedanke unserer Reform ist die Lehre der Wirtschaftswissenschaften wieder mit der Funktionsweise der tatsächlichen Wirtschaft zu verknüpfen“. Damit sagt die task force implizit aus, dass sich die Lehre der Gegenwart von der realen Welt entfernt hat. Zahlreiche deutsche Ökonominnen und Ökonomen unterzeichneten 2016 das Bekenntnis zu einer „Transformativen Wirtschaftswissenschaft" (Schneidewind 2016), die Nachhaltigkeitsziele berücksichtigt. Schneidewind (2016, S. 33) stellt hier die Frage: „Wie kann die Lehre an Universitäten und Hochschulen so gestaltet werden, dass die dort ausgebildeten jungen Menschen in die Lage versetzt werden, die Herausforderungen für eine bessere Zukunft zu bewältigen?" Auch diverse öffentliche Aufrufe für eine Plurale Ökonomik von Studierenden in der ganzen Welt fordern die Thematisierung aktueller und konkreter (politischer) Fragen vor dem Hintergrund der sozialökologischen Krisen unserer Zeit. Zukunftsgestaltung steht auch hier im Vordergrund. Die Bedeutung des „Echtweltbezugs“ findet sich bei einigen Gruppierungen bezeichnenderweise bereits im Titel ${ }^{1}$. Was impliziert dies für die wirtschaftswissenschaftliche Didaktik? Wie kann Diskursfähigkeit gelehrt und „Echtweltbezug“ gewährleistet werden? Während sich aus den Hauptforderungen der Pluralen Ökonomik nach Theorien- und Methodenvielfalt (z. B. ISIPE 2014) unmittelbar inhaltliche Empfehlungen für die Lehre ableiten lassen, und sich vergleichsweise einfach beurteilen lässt, ob ein Lehrvorhaben diesbezüglich erfolgreich ist, scheint die Antwort an dieser Stelle weniger offensichtlich. Der folgende Seminarvorschlag stellt einen Versuch dar, Diskurskultur zu stärken und reale Probleme in das Zentrum der Lehre zu stellen.

Die Veranstaltung wurde im Rahmen des Masters „Plurale Ökonomik“ an der Universität Siegen und folglich vor dem Hintergrund eines pluralistischen Bildungsideals angeboten. Sowohl das Ziel der Kultivierung von Diskursen als auch der Bezug auf reale Problemstellungen verknüpft mit der Bedeutung der

${ }^{1}$ Z. B. beim studentischen Arbeitskreis „Real World Economics Heidelberg“. 
Kontextualisierung von Wirtschaft und wirtschaftlicher Bildung wird von den Siegener Pluralen Ökonominnen und Ökonomen geteilt. Der Master Plurale Ökonomik stellt (insbesondere im Rahmen des organisationalen Schwerpunkts „Management und Mitweltgestaltung") die Rolle von Ökonominnen und Ökonomen als Gestalterinnen und Gestalter in den Vordergrund. Hierzu benötigen Ökonominnen und Ökonomen „Fähigkeiten der Kooperation, der Systementwicklung und der Kreativität“" (Forschungsstelle Plurale Ökonomik 2019).

Der Master Plurale Ökonomik ist ein junger Studiengang ohne langjährig etablierte Strukturen, woraus sich ein relativ hoher Freiheitsgrad für die Lehre ableiten lässt. Konkret wurde das Seminar 2017 und 2018 im ersten Semester als Ergänzung zum einführenden Modul „Plurale Ökonomik“ angeboten. Die Veranstaltung war nicht Teil des offiziellen Modulhandbuchs. Die Teilnahme der jeweiligen Kohorte von etwa 30 Studierenden war folglich freiwillig.

Mein persönlicher Erfahrungskontext war zum Zeitpunkt der Entwicklung des Veranstaltungskonzepts vor allem geprägt von selbstorganisierten, partizipativen Lernformen. Hervorzuheben ist hier insbesondere der studentisch organisierte Lesekreis des Arbeitskreises Plurale Ökonomik an der Universität Leipzig². Um dem Bedarf der Studierenden nach offenen Austauschformaten gerecht zu werden, entstand im Leipziger Lesekreis ein Raum für tiefer gehende Fragen der Teilnehmenden. Ein persönliches Ziel der hier beschriebenen Seminarkonzeption war, ein ähnliches Format an der Universität Siegen zu etablieren. Den Studierenden sollte ermöglicht werden, den Lernraum im vorab definierten Rahmen mitzugestalten. Handelte es sich beim Lesekreis um ein komplett offenes Format, das allen Interessierten offen stand, sollte sich das an der Universität Siegen angebotene Seminar in das entsprechende Modul einfügen, und sah die Teilnahme der Studierenden des Masterstudiengangs Plurale Ökonomik vor.

\footnotetext{
${ }^{2}$ Die Veranstaltung findet seit dem Wintersemester 2013/2014 regelmäßig mit unterschiedlichen Teilnehmendenzahlen als offene Diskussionsrunde statt. Ziel der Initiatorinnen Maike Kilian und Elsa Egerer war es, einen Raum zu schaffen, in dem die Teilnehmenden die Standardlehre diskutieren, hinterfragen und in einen pluralen Kontext stellen können. Der Arbeitskreis Plurale Ökonomik ist als Untergruppe der Leipziger Lokalgruppe von oikos organisiert. Oikos ist eine internationale Studierendenorganisation, die sich für eine nachhaltige Gesellschaft einsetzt.
} 


\section{Innovative Lösung im Sinne einer pluralen, sozioökonomischen Hochschulbildung}

\subsection{Konzept und Ziele}

Im Rahmen des Seminars lernen die Studierenden verschiedene ökonomische Denkrichtungen kennen, welche auf aktuelle wirtschaftspolitische Fragen bezogen werden. Im Rahmen verschiedener strukturierter Formate kommen die Studierenden miteinander ins (Streit-)Gespräch.

Das nachfolgend beschriebene Konzept weist Gemeinsamkeiten mit dem von Nelson (2011) vorgeschlagenen „broader questions and bigger toolbox approach“ auf. Ähnlich wie im hier vorgestellten Seminar umgesetzt, spricht sich Nelson (2011) dafür aus, Fragen der realweltlichen Wirtschaft ins Zentrum der Lehre zu stellen.

Zunächst hat das Seminar das Ziel, die Fachkompetenz der Studierenden auf dem Gebiet der Pluralen Ökonomik weiterzuentwickeln und das Gelernte anzuwenden. Neben der tiefer gehenden inhaltlichen Auseinandersetzung mit verschiedenen ökonomischen Denkrichtungen sollen die Studierenden für den Nutzen einer pluralistischen Herangehensweise sensibilisiert werden. Die Anwendung verschiedener theoretischer Konzepte führt zu einem ganzheitlicheren Bild von Wirtschaft und im Idealfall zu einer Vielfalt unterschiedlicher Lösungsansätze für praktische Probleme.

Durch das Format der strukturierten Diskussion wird die Methodenkompetenz und Reflexionsfähigkeit der Studierenden gestärkt. Indem die Studierenden im Gespräch mit unterschiedlichen Perspektiven konfrontiert werden, werden sie herausgefordert, ihren Standpunkt zu vertreten, diesen argumentativ zu begründen und gegen Gegenargumente zu verteidigen. Dabei können sie auch rhetorische Fertigkeiten entwickeln und (ein-)üben.

Das Seminar kann zudem die Problemlösungskompetenz der Studierenden fördern. Indem theoretische Konzepte auf praktische Fragestellungen angewendet werden, ist eine Transferleistung der Studierenden gefordert, der sie weitgehend autonom und kreativ begegnen können.

Nicht zuletzt soll das Seminar die sozialen Kompetenzen der Studierenden fördern, insbesondere die Kommunikationsfähigkeit. Im Sinne des dialogischen Pluralismus sollen die Studierenden lernen, flexibel und beweglich auf Argumente zu reagieren. Sie sollen befähigt werden, ,verschiedene Perspektiven einzunehmen und im Dialog zu halten“ (Rommel und Schneidewind 2017, S. 24). 


\subsection{Ablauf des Seminars}

\subsubsection{Themen- und Gruppenwahl}

$\mathrm{Zu}$ Beginn des Seminars werden die Studierenden befragt ${ }^{3}$, welche Themenkomplexe sie diskutieren möchten (z. B. Arbeitsmarktpolitik, Umweltpolitik). ${ }^{4}$ Anschließend finden sich die Studierenden in Gruppen ${ }^{5}$ zusammen und wählen gemeinsam eine ökonomische Denkschule (oder eine einzelne Vertreterin bzw. einen einzelnen Vertreter), mit welcher bzw. welchem sie sich vertieft beschäftigen möchten. ${ }^{6}$ Die Literaturbeschaffung ist Aufgabe der Studierenden. Als grobe Orientierung empfiehlt sich z. B. die Online-Lernplattform https://www. exploring-economics.org/de/. Eine breitere Auflistung verschiedener ökonomischer Strömungen findet sich z. B. bei Peukert (2019). Bei der Auswahl der Denkschulen ist darauf zu achten, dass die Perspektiven möglichst heterogen sind. ${ }^{7}$

\footnotetext{
${ }^{3}$ Aufgrund zeitlicher Beschränkung wurden insbesondere im zweiten Durchlauf einzelne Elemente aus dem Seminarkonzept gekürzt. Die folgende Beschreibung geht in Teilen über die umgesetzte Gestaltung hinaus.

${ }^{4}$ Formatvorschlag: Die Studierenden sprechen in Kleingruppen über Themen, die sie interessieren und erstellen hierzu Plakate, die im Plenum vorgestellt werden. Anschließend erhalten die Studierenden Klebepunkte, die sie hinter die Nennungen auf denjenigen Plakaten platzieren, die sie am meisten interessieren. Mithilfe der Klebepunkte können die Interessensschwerpunkte visualisiert werden.

${ }^{5}$ Die Gruppenanzahl orientiert sich an der Zahl der Teilnehmenden und dem zeitlichen Rahmen. Z. B. wurde die Diskussion im zweiten Durchlauf des Seminars (drei Themenblöcke, sechs Gruppen) wie folgt strukturiert. Das Thema „Ungleichheit/Verteilungsfragen“ wurde aus der Perspektive von Marxismus, Neoklassik, Postkeynesianismus, und Feministischer Ökonomik beleuchtet. Der zweite Themenblock „Umweltpolitik“ wurde aus dem Blickwinkel der Schulen Ordoliberalismus, Neoklassik, Feministische Ökonomik sowie Sozioökonomik besprochen. Zum Thema „Digitalisierung“ diskutierten die Studierenden aus der jeweiligen Sichtweise von Ordoliberalismus, Sozioökonomik, Marxismus und Postkeynesianismus. Die genannten Denkschulen wurden zuvor unter anderem in der Vorlesung „Einführung in die Plurale Ökonomik“ besprochen.

${ }^{6}$ Vorschlag für den Medieneinsatz: Den Studierenden kann als Hilfsmittel zur selbstorganisierten Gruppenfindung die Verwendung eines kollaborativen web-basierten real-timetexteditors empfohlen werden (z. B. Etherpad, Googledoc). Hier können die Studierenden selbstständig und für alle sichtbar eintragen, welche Denkschule sie bearbeiten möchten.

${ }^{7}$ Um Heterogenität und Ausgewogenheit zu gewährleisten, kann z. B. darauf geachtet werden, dass sowohl Denkschulen vertreten sind, die dem heterodoxen Spektrum zugeordnet werden, als auch Denkschulen, die als Teil des Mainstreams gelten (z. B. Peukert 2019, S. 4 ff.). Eine weitere Möglichkeit ist die Abdeckung verschiedener Perspektiven hinsichtlich Ontologie, Epistemologie und Methodologie (z. B. Exploring Economics 2019).
} 


\subsubsection{Vorstellung und Reflexion zur der gewählten Denkschule}

Vor Beginn der inhaltlichen Bearbeitung der aktuellen Themenkomplexe betrachten die Studierenden die gewählte Denkschule aus wissenschaftstheoretischer und ideengeschichtlicher Sicht. Hier sollen Fragen nach dem historischen Kontext, Ontologie, Epistemologie und Methodik gestellt werden, z. B.: Wann ist die Schule entstanden? Was waren wichtige geschichtliche Ereignisse dieser Zeit? Wer sind die wichtigsten Vertreterinnen und Vertreter dieser Schule? Welches Menschenbild/Wissenschaftsverständnis wird in ihr zugrunde gelegt? Worauf baut die Schule auf? Von wem/was grenzt sie sich ab? Welche Methoden wendet sie an? ${ }^{8}$ Um das Wissenschaftsverständnis verschiedener Schulen zu visualisieren bietet sich eine Aufstellung an. Die Studierenden werden hierfür aufgefordert, ,ihre' Schule im Raum zwischen zwei (wissenschaftstheoretischen) Polen (z. B. gemäß Marietta und Perlman 2000 oder Peukert 2018) zu verorten. Beispielfragen können dabei lauten: Blickt Ihre Schule eher aus der Perspektive des methodologischen Individualismus oder eher aus der Perspektive des methodologischen Holismus auf eine Problemstellung? Geht Ihre Schule eher formalistisch oder verbal-argumentativ vor? Würden Sie das Wissenschaftsverständnis Ihrer Schule eher dem Falsifikationismus oder dem Fallibilismus zuordnen?

\subsubsection{Vorstellung der verschiedenen Denkschulperspektiven auf das gewählte Thema}

$\mathrm{Zu}$ jedem Themenkomplex folgen zunächst, geordnete" Inhaltssessions. Hier stellen sich die Studierenden gegenseitig (nacheinander) die Perspektive ,ihrer Schule auf das Themenfeld vor. Zu Beginn soll geklärt werden, was die jeweilige Denkrichtung unter den themenbezogenen Begrifflichkeiten versteht und welche Prämissen vorausgesetzt werden. Auf dieser Grundlage ist es Aufgabe der Studierenden, die erarbeiteten Texte auf wirtschaftspolitische Fragen zu beziehen und - sofern möglich - Gestaltungsvorschläge abzuleiten. Finden sich bereits in der Literatur (der jeweiligen Schule) Politikmaßnahmen, sind die Quellen zu benennen. Handelt es sich um eine freiere Interpretation der Studierenden, ist

\footnotetext{
${ }^{8}$ An dieser Stelle sollte vonseiten der Lehrenden darauf hinweisen werden, dass die Abgrenzung verschiedener Denkschulen je nach Quelle ggf. unterschiedlich gezogen wird. Auch die Beantwortung der genannten Fragen lässt sich häufig nicht für alle Vertreter einer Denkschule einheitlich definieren. Die Studierenden sollten dafür sensibilisiert werden, dass das Denken in Kategorien (hier Denkschulen) in den Sozialwissenschaften immer eine vereinfachte Betrachtungsweise darstellt.
} 
diese entsprechend zu begründen. Existieren innerhalb der Schule unterschiedliche Auffassungen zum Thema, ist dies ebenfalls zu erörtern.

\subsubsection{Die verschiedenen Denkschulperspektiven im Diskurs}

Die Diskussionstermine bilden das Herz des Seminars. Hier kommen die Studierenden miteinander ins offene (Streit-)Gespräch. Der Diskussionsraum gliedert sich in eine Podiumsdiskussion, eine offene Fishbowl-Diskussion und einen Feedbackteil.

Bei der Podiumsdiskussion werden konkrete wirtschaftspolitische Empfehlungen (z. B. aus Parteiprogrammen) zum Themenfeld diskutiert. Die Diskussion wird von einem Moderatorenteam oder der Lehrperson vorbereitet und wird ähnlich einer politischen Talkshow gestaltet. Hierfür bereitet die Moderation (kritische) Fragen vor, die von drei bis vier Podiumsteilnehmerinnen und Podiumsteilnehmern im Diskurs beantwortet werden. Die Studierenden übernehmen dabei die Rolle einer Vertreterin oder eines Vertreters, ihrer" Schule und argumentieren aus dieser Perspektive problembezogen. Als Einstieg bietet sich ein ,Faktencheck“ mit empirischen Daten an, welcher vom Moderatorenteam erarbeitet wird. Im Anschluss an die Podiumsdiskussion folgt ein Debriefing. Hier sollen die Studierenden berichten, wie sie ihre Rolle als Expertinnen und Experten der jeweiligen Schule wahrgenommen haben und Fragen beantworten wie: Wo konnten Sie in Ihrer Rolle gut argumentieren? Wo ist es Ihnen schwer gefallen? Wie stehen Sie selbst zu den vorgebrachten Argumenten?

In der folgenden offenen Fishbowl-Diskussion diskutieren die Studierenden frei zum Thema und können hierbei ihren eigenen Standpunkt vertreten. Hierzu werden in der Mitte des Raums drei bis vier Stühle im Kreis platziert. Als Regel wird vereinbart, dass nur die Studierenden sprechen, welche auf einem der Stühle in der Fishbowl sitzen. Wer etwas zur Diskussion beitragen möchte, muss folglich zunächst in der Fishbowl Platz nehmen. Hierzu können sich Studierende auf einen (ggf.) freien Stuhl setzen oder den Platz von anderen Diskussionsteilnehmenden einnehmen. Vorab wird die Regel vereinbart, dass im letzteren Fall eine Person durch Antippen am Rücken aus der Runde gewählt wird.

\section{$3 \quad$ Folgen/Wirkungen}

Im Folgenden soll reflektiert werden, welche Herausforderungen der dialogische Pluralismus an die Gesprächsteilnehmenden stellt und inwiefern das Seminar die Studierenden in ihrer Diskursfähigkeit stärken kann. Zudem wird grundsätzlicher reflektiert, wie sich die Rolle der Lehrenden bei partizipativ gestalteten Seminaren verändert. 


\subsection{Ein geeignetes Format hilft, den Gesprächsfaden zu halten}

Ein offener Diskussionsraum macht noch keine gute Diskussion. Nach meiner Erfahrung fehlt in vielen Diskussionen die Fähigkeit oder die Bereitschaft, an einem gemeinsamen Denkstrang zu arbeiten und die Potenziale der Konversation werden nicht ausgeschöpft. Je größer die Anzahl der Gesprächsteilnehmenden, desto höher ist die Wahrscheinlichkeit, dass sich das Gespräch in Einzelstatements ohne Bezug zueinander verliert. Obgleich auch im Rahmen des Seminars ein derartiges Zerfasern des Gesprächs an einzelnen Stellen zu beobachten war, konnte das Gespräch durch die klaren Formatvorgaben sinnvoll eingerahmt werden. Z. B. wurde die Anzahl der Diskussionsteilnehmenden durch das Format der Podiumsdiskussion und der Fishbowl begrenzt. Zusätzlich konnte das Gespräch durch die Moderation gelenkt werden.

Die Fishbowl besticht durch ihre klare Struktur und die Möglichkeit der Selbstmoderation durch die Studierenden. Gleichzeitig können alle Studierenden an der Diskussion teilnehmen und sich spontan einbringen. Gleichwohl lief im Rahmen des Seminars auch manche Fishbowl-Diskussion teilweise schleppend an und gestaltete sich, sofern nicht moderiert wurde ${ }^{9}$, ergebnisarm.

Die (weniger freien) Podiumsdiskussionen im Rahmen des Seminars verliefen sehr produktiv. Einige Studierende berichteten im Nachhinein beispielsweise, dass sie nun ein besseres Verständnis davon hätten, was Plurale Ökonomik eigentlich bedeute. Insbesondere die nachfolgend beschriebenen Aspekte schienen für das Gelingen von Relevanz.

\subsection{Gelingensbedingungen der Podiumsdiskussion}

Die Moderation der Podiumsdiskussion war für den Ablauf des Gesprächs entscheidend. Bei der Planung des Gesprächs war es für ein gutes Streitgespräch erforderlich, die Denkschulen so auf die Themen aufzuteilen, dass sich in der Diskussion unterschiedliche Perspektiven aufzeigen lassen, sowie darauf zu achten, dass jede Denkschule zum Thema ,etwas zu sagen hat'. Dabei war es insbesondere herausfordernd, eine Mischung aus breiten und spezifischen

\footnotetext{
${ }^{9}$ Die Methode Fishbowl kann dahin gehend angepasst werden, dass eine Moderatorin bzw. ein Moderator teilnimmt.
} 
Fragen zu finden. Die Fragen sollten breit und offen genug sein, um eine Bezugnahme auf verschiedene Theorien zu ermöglichen. Diverse Ökonominnen und Ökonomen verschiedenster Theorien äußern sich z. B. zu Fragestellungen zum breit formulierten Thema „Soziale Absicherung“, weniger Ökonomen und Ökonominnen beschäftigen sich mit konkreten Politikvorschlägen. Eine breite Themenstellung erleichtert es den Studierenden folglich, etwas aus der Perspektive einer Schule beizutragen. Gleichzeitig birgt eine breite Themenstellung die Problematik, dass oberflächlicher bzw. weniger anwendungsbezogen diskutiert wird. Bezugnehmend auf das Seminar lässt sich sagen, dass das Thema vorab stärker hätte eingegrenzt werden müssen, um eine tiefer gehende und stärker auf konkrete Maßnahmen bezogene Diskussion zu erreichen. Auf der anderen Seite konnten durch die Formulierung breiterer Fragen alle Studierenden (und Denkschulen) erfolgreich eingebunden werden. ${ }^{10}$ Die Bereitschaft der Studierenden, sich intensiv auf das Seminar vorzubereiten, ist in diesem Zusammenhang als weitere Gelingensbedingung zu nennen.

Meines Erachtens ausschlaggebend für die gute Diskussionsdynamik war die Konzeption als Rollenspiel. Rollenspiele bieten die Möglichkeit, neue Verhaltens- und Argumentationsmöglichkeiten im künstlichen Rahmen zu erproben. Durch das Rollenspiel kann eine emotionale Distanz zur eigenen Person geschaffen werden und gleichzeitig wird die Fähigkeit gefördert, sich in ,fremde Perspektiven und Denkweisen hineinzuversetzen und deren Beweggründe zu verstehen (Perspektivenübernahme). Über den spielerischen Zugang, in die Rolle einer Expertin oder eines Experten zu schlüpfen, entwickelten die Studierenden große Diskussionsfreude. So wurden aus vormals wortkargen, zurückhaltenden Studierenden streitbare Gesprächsteilnehmerinnen und Gesprächsteilnehmer. Meines Erachtens ist dies auch darauf zurückzuführen, dass Angst vor möglicherweise negativ konnotierten oder vermeintlich disharmonischem Verhalten abgebaut werden konnte, da die Studierenden in ihrer Rolle agierten und das Verhalten nicht ihrer Person zugeschrieben wurde. Während in anderen Diskussionen, auch aufgrund der hohen Homogenität der ökonomischen Urteile der

\footnotetext{
${ }^{10}$ Zur Veranschaulichung sind im Folgenden einige breitere Beispielfragen aus der Podiumsdiskussion zum Thema (Um-)Verteilung angeführt: Wie kommt es zu Ungleichheit? Ist eine ungleiche Verteilung als problematisch zu beurteilen? Wie beurteilen Sie die gegenwärtige Lage? Welche Aspekte sind wichtig/sollten intensiver diskutiert werden? Sollte der Staat eingreifen? Wieso (nicht)? Wenn ja, mit welchen politischen Maßnahmen? Sollten Menschen nach ihrer Leistung entlohnt werden? Ist dies gegenwärtig der Fall? Besteht ein Spannungsfeld zwischen Wirtschaftswachstum und Umverteilungsmechanismen?
} 
Studierenden, häufig die Reibungsfläche fehlte, konnten durch die Konstellation der Podiumsdiskussion Gegensätze aufgezeigt und ausdiskutiert werden.

Ein weiteres Ziel des Seminarkonzepts ist es, die Kommunikationsfähigkeit der Studierenden zu fördern, indem sie nachvollziehen, dass Differenzen zwischen verschiedenen Perspektiven häufig auf (verborgene) unterschiedliche Grundannahmen zurückzuführen sind. Ob dies gelungen ist, lässt sich abschließend nicht sagen, unter anderem weil in beiden Durchläufen des Seminars die wissenschaftstheoretische Reflexion aus zeitlichen Gründen gekürzt werden musste.

\subsection{Sozialkompetenz als Vorbedingung für dialogischen Pluralismus}

Resümierend lässt sich sagen, dass dialogischer Pluralismus insbesondere die Entwicklung von Sozialkompetenzen erfordert. Offene, innovative Formate, die den Studierenden die Möglichkeit geben, sich auszuprobieren, sind unter diesem Aspekt sinnvoll. Dabei geht es meines Erachtens auch darum, Räume zu schaffen, in denen eine begrenzte Autonomie der Studierenden möglich ist. Das Motto,je offener desto besser", ist jedoch nicht tragfähig. Denn auch wenn sich einzelne Studierende einen ,herrschaftsfreien“ Raum wünschen, zeigt sich häufig, dass eine klare Struktur und Moderation dem Gruppeninteresse an einem produktiven Diskussionsraum zuträglicher sind. Die Fruchtbarkeit eines Gesprächs hängt darüber hinaus stark vom Entwicklungsstand der Gruppe ab. Das vorgestellte Diskussionsseminar profitierte in beiden Durchläufen an der Universität Siegen von einer positiven Dynamik innerhalb der Gruppe der Studierenden.

Der Einfluss eines einzelnen Seminars auf die Gesprächskultur ist begrenzt. Im besten Fall können Strukturen geschaffen werden, durch die Studierende angeregt werden, ihre Persönlichkeit zu entfalten und Sozialkompetenz (weiter) zu entwickeln. Die Frage, wie auf das mangelnde Selbstbewusstsein von einzelnen Gesprächsteilnehmenden eingegangen werden kann, bleibt für mich offen. Ein Impuls zur Selbstreflexion wurde gesetzt, indem die Studierenden angeregt wurden, Mitverantwortung für die Gruppenkommunikation zu übernehmen und das eigene Gesprächsverhalten mithilfe eines Fragebogens ${ }^{11}$ zu reflektieren.

\footnotetext{
${ }^{11}$ Der Fragebogen wird bei Interesse gerne zur Verfügung gestellt.
} 


\subsection{Die Rolle des Lehrenden bei partizipativ gestalteten Seminaren}

Meine Erfahrungen, auch aus anderen Veranstaltungen, haben mich darin bestärkt - gerade bei innovativen Konzepten - darauf hinzuweisen, dass partizipative Mitgestaltung der Lehre nicht basisdemokratische Entscheidung bedeutet. Die Verantwortung für und Macht über den Seminarrahmen liegt immer bei der Lehrperson. Um hier potenzielle Enttäuschungen aufseiten der Studierenden zu vermeiden, ist es meines Erachtens hilfreich, auf diesen Aspekt hinzuweisen.

Je freier die Gestaltung eines Seminars, desto stärker drängt sich die Frage des beruflichen Selbstbildes der Lehrperson und die Notwendigkeit, den Studierenden die eigene Rolle zu kommunizieren, auf. Dies äußert sich auf zwei Ebenen: Einerseits ist die Lehrperson verantwortlich für die Gestaltung des Seminarraums. Auch wenn die Lehrperson sich im Rahmen einer Diskussion nicht in den Vordergrund drängen möchte, bleibt ihr als Aufgabe mindestens, ,den Raum zu halten” (Polylog 2017). Das heißt, die Lehrperson stellt sicher, dass der Rahmen, in dem eine Diskussion stattfindet, vorab definiert und den Studierenden bekannt ist. Zudem öffnet und schließt die Lehrperson die Diskussion. Wird die Moderationsrolle an die Studierenden oder die Gruppe abgegeben, ist es für den Erfolg eines Seminars erforderlich, dies klar zu kommunizieren.

Des Weiteren stellt sich die Frage des beruflichen Selbstbildes auf inhaltlicher Ebene. Je offener und damit potenziell umfangreicher der Seminarfokus, desto größer die inhaltlichen Anforderungen an die Lehrperson. Bei einer sehr freien Seminargestaltung, die z. B. transdisziplinär angelegt ist, kann die Lehrperson nicht in allen Bereichen als Expertin oder Experte auftreten. Es besteht folglich ein Zielkonflikt zwischen dem Freiheitsgrad der Seminargestaltung und der Sicherstellung der Korrektheit der diskutierten Informationen. Ein nicht deterministisches Lehrideal führt nicht zur Auflösung dieses Zielkonflikts - im Gegenteil. Laut Earl (2000) zielt ein nicht deterministisches Lehrideal im Gegensatz zur Vermittlung der einen vermeintlich, richtigen“ Theorie darauf $\mathrm{ab}$, den Lernprozess der Studierenden dahin gehend zu unterstützen, dass im Rahmen eines unterstützenden ,Gerüsts“ eigene Schlussfolgerungen gezogen werden können. Während Sicherstellung der Korrektheit im Sinne eines deterministischen Lehrideals bedeutet, zu überprüfen, ob die Studierenden das Problem ,richtig' gelöst haben, birgt das nicht deterministische Lehrideal im beschriebenen Seminarkontext die Herausforderung, zu bewerten, ob 1) die Argumentation verschiedener Theorieansätze korrekt verstanden und wiedergegeben wurde, ob 2) verschiedene Theorien schlüssig angewendet wurden 
und, ob 3) die empirische Datengrundlage sowie der rahmengebende Status quo (z. B. Steuersätze, Sozialgesetzgebung etc.), auf den in der Anwendung Bezug genommen wird, korrekt dargestellt wurden. Je konkreter und detaillierter diskutiert wird, desto herausfordernder ist es für die Lehrperson, die inhaltliche Qualität sicherzustellen.

\section{Literatur}

Bündnis Transformative Lehre Halle (2017). Positionspapier für eine transformative Lehre an der Martin-Luther-Universität Halle-Wittenberg. https://blogs.urz.uni-halle.de/ pluraleoekonomik/files/2017/11/PoPaPi-Transformative-Lehre.pdf. Zugegriffen: 20. Sept. 2019.

Dobusch, L., \& Kapeller, J. (2012). Heterodox United vs. Mainstream City? Sketching a framework for interested pluralism in Economics. Journal of Economic Issues, 4, 10351058.

Earl, P. E. (2000). Indeterminacy in the Economics classroom. In P. E. Earl \& S.F. Frowen (Hrsg.), Economics as an Art of Thought: Essays in Memory of G.L.S. Shackle (S. 25-50). London: Routledge.

Exploring Economics (2019). Orientieren. https://www.exploring-economics.org/de/ orientieren/\#compare. Zugegriffen: 20. Sept. 2019.

Forschungsstelle Plurale Ökonomik an der Universität Siegen (2019). Unser Pluralismusverständnis. https://plurale-oekonomik-siegen.de/forschungsstelle/. Zugegriffen: 20. Sept. 2019.

Gräbner, C., \& Strunk, B. (2018). Pluralism in economics: its critiques and their lessons. ICAE Working Paper Series No. 82. Linz: Institute for Comprehensive Analysis of the Economy (ICAE). https://www.jku.at/fileadmin/gruppen/108/ICAE_Working_Papers/ wp82.pdf. Zugegriffen: 23. Dez. 2019.

INET (2010). Economics of the Real World - A project of the INET Economics Curriculum Task Force. December 2010. Online: https://www.ineteconomics.org/uploads/downloads/Economics_of_the_Real_World_v10.pdf. Zugegriffen: 20. Sept. 2019.

ISIPE (2014). Internationaler studentischer Aufruf für eine Plurale Ökonomik. Online: http://www.isipe.net/home-de. Zugegriffen: 20. Sept. 2019.

Krueger, A., Arrow, K., Blanchard, O., Blinder, A., Goldin, C., Leamer, E., Lucas, R., Panzar, J., Penner, R., Schultz, T. P., Stiglitz, J., \& Summers, L. (1991). Report of the Commission on Graduate Education in Economics. Journal of Economic Literature, 29, 1035-1053.

Marietta, M., \& Perlman, M. (2000). The Uses of Authority in Economics: Shared Intellectual Frameworks as the Foundation of Personal Persuasion. The American Journal of Economics and Sociology, 59, 151-189.

McCloskey, D. (1983). The Rhetoric of Economics. Journal of Economic Literature, 21, 481-517. 
Nelson, J. A. (2011). Broader questions and a bigger toolbox: A problem-centered and student-centered approach to teaching pluralist economics. Real World Economics Review 58, 9-18.

Peukert H. (2019). Eine kritische Analyse an deutschen Hochschulen vorherrschender Einführungen in die Mikro- und Makroökonomie und plural-heterodoxe Alternativlehrbücher. Forschungsinstitut für Gesellschaftliche Weiterentwicklung (FGW): Düsseldorf. FGW-Publikation Neues ökonomisches Denken 09. http://www.fgw-nrw.de/fileadmin/ user_upload/NOED-Studie-09-Peukert-2019_01_29-komplett-web.pdf. Zugegriffen: 23. Dez. 2019.

Plurale Ökonomik Mannheim e.V. https://www.pluraleoekonomik-mannheim.de/. Zugegriffen: 23. Dez. 2019.

Polylog (2017). Diskussion im Rahmen des Workshops “Polylog! Wie reden wir?”. Herbstdialoge 2017 der Cusanus Hochschule.

Rommel F. (2017). Wegkunde einer pluralen Methodik - Wie lässt sich Methodenvielfalt in den Wirtschaftswissenschaften denken? Gehalten am: Eröffnungsvortrag des Moduls "Methodenpluralismus" an der Universität Siegen. Zugegriffen: 19. Mai 2017.

Rommel, F., \& Schneidewind, U. (2017). Auseinandersetzung mit der Kritik an transformativer und pluraler Ökonomik. Ökologisches Wirtschaften, 32, 22-24.

Schneidewind, U. (2016). Transformative Wirtschaftswissenschaft im Kontext nachhaltiger Entwicklung. Ökologisches Wirtschaften, 31, 30-34.

Open Access Dieses Kapitel wird unter der Creative Commons Namensnennung 4.0 International Lizenz (http://creativecommons.org/licenses/by/4.0/deed.de) veröffentlicht, welche die Nutzung, Vervielfältigung, Bearbeitung, Verbreitung und Wiedergabe in jeglichem Medium und Format erlaubt, sofern Sie den/die ursprünglichen Autor(en) und die Quelle ordnungsgemäß nennen, einen Link zur Creative Commons Lizenz beifügen und angeben, ob Änderungen vorgenommen wurden.

Die in diesem Kapitel enthaltenen Bilder und sonstiges Drittmaterial unterliegen ebenfalls der genannten Creative Commons Lizenz, sofern sich aus der Abbildungslegende nichts anderes ergibt. Sofern das betreffende Material nicht unter der genannten Creative Commons Lizenz steht und die betreffende Handlung nicht nach gesetzlichen Vorschriften erlaubt ist, ist für die oben aufgeführten Weiterverwendungen des Materials die Einwilligung des jeweiligen Rechteinhabers einzuholen.

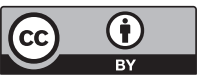

